\title{
S.P.Q.R. Legged Team
}

\author{
D. Nardi, V. Bonifaci, C. Castelpietra, U. Di Iorio, A. Guidotti, L. Iocchi, \\ M. Salerno, and F. Zonfrilli \\ Dipartimento di Informatica e Sistemistica \\ Universitá di Roma "La Sapienza" \\ Via Salaria 113 - 00198 Roma, Italy \\ last_name@dis.uniroma1.it, \\ http://www.dis.uniroma1.it/ leggedro/
}

\begin{abstract}
The SPQR (Soccer Player Quadruped Robots, but also Senatus PopolusQue Romanus) team participated for the second time to Sony Legged League in RoboCup 2001. This work is a team description where it will be highlighted what the team development effort focused on: the realization of a motion module, the realization of a vision module and the improvement of the plans which characterize the different robot roles.
\end{abstract}

\section{Introduction}

S.P.Q.R. is the group of the Faculty of Engineering at University of Rome "La Sapienza" in Italy, that is currently involved in developing two RoboCup teams: the S.P.Q.R. Legged team [7] in the Sony Legged League and the S.P.Q.R. Wheeled team in the Middle Size League. Compared to the team which participated to RoboCup 2000, the team was substantially improved. In particular we have developed our own locomotion, thus abandoning the usage of the OMoNet module [4], which supervises the generation of movement commands, and our own object recognition module in substitution of the Sony's object recognition module. We also introduced localization for our robots. Moreover a great effort was involved in porting the software developed for the previous version of the Sony Aibo robotic platform to the new one which became available on April 2001.

\section{Team Development}

Team Leader: Daniele Nardi

Team Members: Luigia Carlucci Aiello, Luca Iocchi, Alessandro De Luca, Giuseppe Oriolo, Vincenzo Bonifaci, Claudio Castelpietra, Ugo Di Iorio, Alice Guidotti, Massimiliano Salerno, Fabio Zonfrilli

Sponsors: Facoltà di Ingegneria, University "La Sapienza" and NETikos Web Mobility.

Country: Italy

A. Birk, S. Coradeschi, and S. Tadokoro (Eds.): RoboCup 2001, LNAI 2377, pp. 717-720 2002.

(C) Springer-Verlag Berlin Heidelberg 2002 


\section{Architecture}

The system relies on a two layer hybrid architecture [1 in order to integrate both deliberative and reactive capabilities [3. The module named Perception

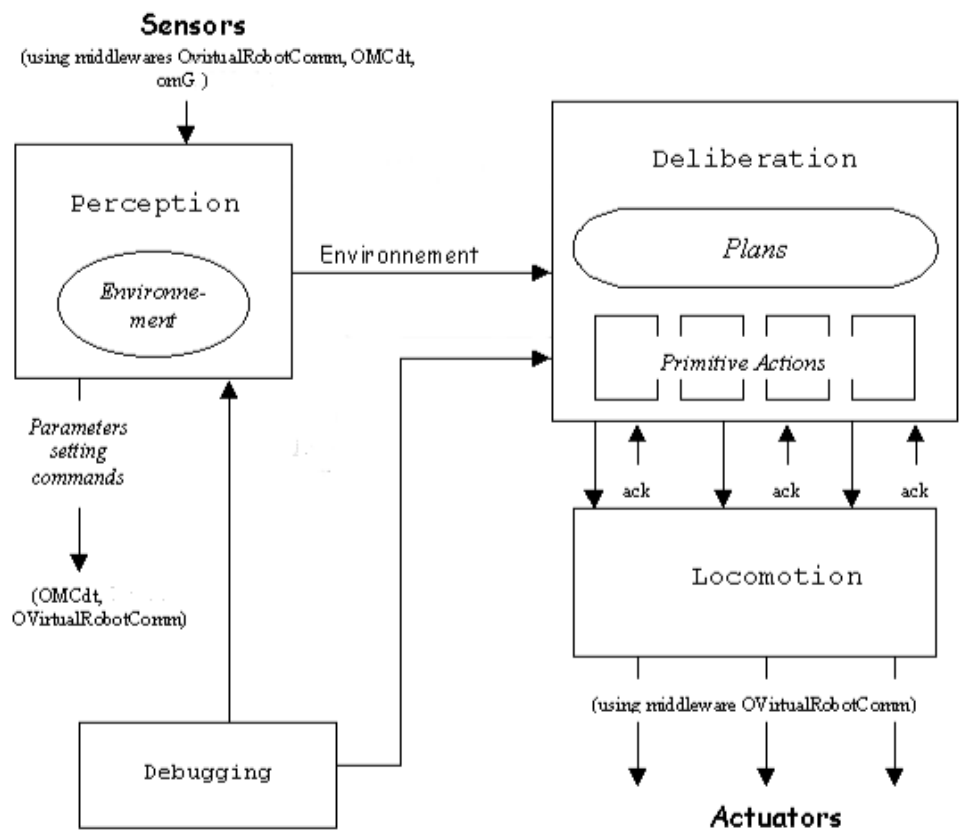

Fig. 1. Architecture

analyzes the sensor data (which are mainly obtained by the camera) and stores and updates the internal world model. The module Deliberation, consisting of a plan execution monitor and a set of primitive actions, receives the world model from Perception, controls the plan of actions to be executed, and generates a set of command for the module Locomotion. The latter provides an interface to the physical functionalities of robot by accepting abstract command from Deliberation and translating them into effective commands to the actuator. The two-level layered architecture is based on different representation of the information: an high-level symbolic one, and a low-level numerical one. The high-level plans are executing depending on the validity of abstract conditions [2], such as KnownBallPosition or NearBall, while low-level atomic actions allow for an immediate reaction to unexpected situations.

\section{Vision}

We have developed our own software for the blob formation and object recognition phases, while for image segmentation we have relied upon the robot hard- 
ware segmentation with the Sony's libraries. This allowed the recognition of objects to be much more stable and provided a solid basis for the design of a localization system. The blob formation routines are based on a RLE compression of the segmented image as described in [5]. This approach has the advantage of being fast and also allows the segmented image to be quickly transmitted through the serial cable to the personal computer for debugging purposes. The object recognition routine takes the blobs and classifies them on the basis of their color, while trying to recover from possibly wrong decisions through the use of some heuristics, depending on the Sony Legged League operational setting. These heuristics make the system less error-prone than before.

\section{Localization}

Building on the capabilities of the vision system, a localization procedure was written, based on trilateration (see [6]). This technique requires the distances and angles of the robot from two known points. Trilateration has the advantage that it does not necessarily require the robot to execute a particular procedure to recognize the markers, thus it allows the robot to extract the information needed without interrupting the task it is accomplishing, but simply by analyzing the acquired images. On the contrary, this technique needs two markers to be recently recognized and therefore the localization information is not always available, thus a reliability value, which is decreasing with time when the markers are not recognized, has been introduced to deal with the robot location.

\section{Behaviors}

The architecture of the deliberative module is based on the one described in [7]. Thus the development effort focused on the primitive action reorganization, in order to make them more parametric (with respect to speed, for instance). Since localization information has been made available, the higher level plans were rewritten in order to take advantage of the knowledge about the robot location. In particular, the goalkeeper can reposition itself on the goal line without having to turn back and look at it (which has proved to be a very risky action). Moreover, localization allowed for a safer and more appropriate choice of the kick to be activated by the robot when it is near the ball, for instance by choosing a right head kick when it knows the opponent goal being on its right, without the need to look at it.

\section{Perception}

The perception module was modified so that the new information needed to localize could be obtained. Moreover, a method, based on the robot kinematic model, was adopted to calculate the position of the robot camera with respect to the ground and thus obtain the ball position relative to the robot. 


\section{Locomotion}

The Sony's OMoNet [4 module has been abandoned in favor of our own motion control. The new Locomotion allowed the robots to increase the motion speed and to have a larger selection of different movements to be used during the task execution. New walking styles and kicks were created through an off-line tool. The kicks developed include: a frontal head kick, lateral head kicks, a leg kick and a chest kick, the latter specific to the goalkeeper. Moreover many types of walking styles were developed so that both more stable and faster movements were obtained, and ad hoc movements for special robot behaviors were made available. The movement speed was increased by using a spline interpolation to smooth the joint control laws, thus avoiding discontinuities in the joint velocities.

\section{$9 \quad$ Special Team Features and Conclusion}

The main focus of the team work has been the realization of a team of cognitive soccer robots by following the design methodology presented in 2], whose main features are a hybrid architecture that is heterogeneous and asynchronous allowing for an effective integration of reasoning and reactive capabilities, a high level representation of the plans executed by the player, a formal account of the system for generating and verifying plans. The architecture proposed has been designed to consent the use of the high level representation and deliberation on both the SPQR Legged Team and the SPQR Wheeled Team. In the future the work will focus on the improvement of the motion, the vision and the localization modules, but mainly on communication based robot coordination, which has been proven to be a central point to obtain effective results.

\section{References}

1. Iocchi, L.: Design and Development of Cognitive Robots. PhD thesis. Dipartimento di Informatica e Sistemistica, Universitadi Roma "La Sapienza". (1999)

2. Castelpietra, C., Iocchi, L., Nardi, D., Rosati, R.: Design and Implementation of Cognitive Soccer Robots. In Procs. of The RoboCup 2001 International Symposium. (2001)

3. Saffiotti, A.:Some Notes on the Integration of Planning and Reactivity in Autonomous Mobile Robots. In Procs. of the AAAI Spring Symposium on Foundations of Automatic Planning. Stanford, CA. (1993) 122-126

4. Sony Corporation: OPEN-R Software Programmer's Guide. (2001)

5. Bruce, J., Balch, T., Veloso, M.: Fast and Cheap Color Image Segmentation for Interactive Robots. In Procs. of IROS-2000. Japan. (2000)

6. Dalgliesh, J., Lawther, M.: Playing soccer with quadruped robots. School of Computer Science and Engineering, University of New South Wales. (1999)

7. Nardi, D., Castelpietra, C., Guidotti, A., Salerno, M., Sanitati, C.: S.P.Q.R. in RoboCup-2000: Robot Soccer World Cup IV. Springer Verlag. (2000) 